Mathematical Modelling AND ANALysis

Volume 18 Number 2, April 2013, 219-235

http://dx.doi.org/10.3846/13926292.2013.781069

(c) Vilnius Gediminas Technical University, 2013
Publisher: Taylor\&Francis and VGTU

http://www.tandfonline.com/TMMA

Print ISSN: 1392-6292

Online ISSN: 1648-3510

\title{
On the Properties of a Class of Polyharmonic Functions
}

\section{Suheil Khuri}

\author{
American University of Sharjah \\ Sharjah, United Arab Emirates \\ E-mail: skhoury@aus.edu
}

Received March 30, 2012; revised January 16, 2013; published online April 1, 2013

\begin{abstract}
The aim of this paper is to investigate some motivated geometrical aspects and properties of polyharmonic functions $(\mathcal{P H})$ including starlikeness, convexity and univalence. A polyharmonicity preserving complex operator is also introduced. Further, a new subclass of polyharmonic functions $(\mathcal{C P H})$ is defined and certain characteristics of elements of this subclass are examined and obtained. In particular, we extend Landau's theorem to functions in this subclass, and consider the GoodmanSaff conjecture and prove that the conjecture is true for mappings belonging to $\mathcal{C P H}$.
\end{abstract}

Keywords: polyharmonic function, univalent, starlike, Landau's Theorem.

AMS Subject Classification: $30 \mathrm{C} 45 ; 31 \mathrm{~B} 30$.

\section{Introduction}

Complex-valued harmonic functions that are univalent and sense preserving in the unit disk $U$ can be written in the form $f=g+\bar{h}$, where $h$ and $g$ are analytic in $U . \mathrm{A}^{2}$ continuous complex-valued function $F=u+i v$ in a domain $D \subset C$ is biharmonic if the Laplacian of $F$ is harmonic, that is $\Delta F$ is harmonic in $D$ if $F$ satisfies the biharmonic equation $\Delta(\Delta F)=0$, where $\Delta=4 \frac{\partial^{2}}{\partial z \partial \bar{z}}$ is the Laplacian operator. The class of biharmonic functions includes the class of harmonic functions and is a subclass of the class of polyharmonic functions. More generally, a $2 p$-continuously differentiable complex-valued function $F=$ $u+i v$ in a domain $D \subset C$ is $p$-harmonic (polyharmonic) if $F$ satisfies the $p$-harmonic equation

$$
\Delta^{p} F:=\Delta \Delta^{p-1} F=0 .
$$

When $p=1$ the mapping is basically harmonic, while the case $p=2$ yields the biharmonic mapping. If $F$ is a biharmonic function then it has the representation

$$
F=r^{2} G+H=|z|^{2} G+H
$$


where $G(z)$ and $H(z)$ are complex-valued harmonic functions in $D$. Throughout this paper we consider $p$-harmonic mappings of the unit disk $U=\{z \in C$ : $|z|<1\}$.

Concerning $p$-harmonic mappings, we have the following characterization which is crucial in our investigations (see [8]).

Proposition. If $F$ is a p-harmonic function in a stardomain D with center 0 then it has the representation:

$$
F=F(z, \bar{z})=\sum_{n=0}^{p-1} r^{2 n} F_{n},
$$

where $F_{n}(z)$ 's are complex-valued harmonic functions in $D$, and $r^{2 n}=|z|^{2 n}=$ $(z \bar{z})^{n}$.

A harmonic function $F$ is locally univalent if the Jacobian of $F, J_{F}$,

$$
J_{F}=\left|F_{z}\right|^{2}-\left|F_{\bar{z}}\right|^{2} \neq 0 .
$$

A function $F$ is orientation preserving if

$$
J_{F}=\left|F_{z}\right|^{2}-\left|F_{\bar{z}}\right|^{2}>0 .
$$

A set $E \subset C$ is said to be starlike with respect to a point $w_{0} \in E$ if and only if the linear segment joining $w_{0}$ to every other point $w \in E$ lies entirely in $E$, while a set $E$ is said to be convex if and only if it is starlike with respect to each of its points, that is, if and only if the linear segment joining any two points of $E$ lies entirely in $E$. A sense-preserving harmonic mapping $f$ is said to be starlike if its range is starlike with respect to the origin. In other words, if some point $w_{0}=f\left(z_{0}\right)$ is in the range of $f$, then so is the entire radial segment from 0 to $w_{0}$. If $f$ has a smooth extension to the closed disk, an equivalent requirement is that $\arg \left\{f\left(e^{i \theta}\right)\right\}$ be a nondecreasing function of $\theta$, or that $\frac{d}{d \theta} \arg \left\{f\left(e^{i \theta}\right)\right\} \geq 0$. For analytic function $f$, this condition takes the form $\operatorname{Re}\left\{\frac{z f^{\prime}(z)}{f(z)}\right\}>0$ for $z \in U$. We say that a univalent polyharmonic (harmonic) function $F$, with $F(0)=0$, is starlike if the curve $F\left(r e^{i t}\right)$ is starlike with respect to the origin for each $0<r<1$. In other words, $F$ is starlike if $\frac{\partial \arg F\left(r e^{i t}\right)}{\partial t}=\operatorname{Re} \frac{z F_{z}-\bar{z} F_{\bar{z}}}{F}>0$ for $z \neq 0$. For accuracy, it is worth noting that $p$-harmonic function is a function of $z$ and $\bar{z}$, that is, $F=F(z, \bar{z})$ and therefore $F=F\left(r e^{i t}, r e^{-i t}\right)$.

A univalent polyharmonic (harmonic) function, $F$, with $F(0)=0$ and also $\frac{\partial F\left(r e^{i t}\right)}{\partial t} \neq 0$ whenever $0<r<1$, is said to be convex if the curve $F\left(r e^{i t}\right)$ is convex for each $0<r<1$. In other words, $F$ is convex if $\frac{\partial \arg \frac{\partial}{\partial t} F\left(r e^{i t}\right)}{\partial t}>0$ for $z \neq 0$.

This paper is motivated by the recent work and development on the subject of biharmonic functions $[1,2,3,4,5,6,13,16]$. These are solutions to the biharmonic equation that arises in physical applications including linear elasticity theory, Stokes flow and radar imaging problems (see [12] and the references within). The purpose of this article is to extend such results to a class of polyharmonic functions (see $[14,17]$ ); some characteristics and geometrically 
motivated properties related to starlikeness, convexity and univalence are examined. A complex operator that preserves polyharmonicity is introduced. Furthermore, we generalize Landau's theorem to functions belonging to this class and show that the Goodman-Saff conjecture $[6,18]$ is valid for such functions as well. Recently Landau's theorem has been extended to biharmonic mappings [1], for planar $p$-harmonic mappings [7], as well as to log- $p$-harmonic mappings [15]. For more details on harmonic mappings and the various definitions introduced see $[9,10,11]$.

\section{Properties of the Class $\mathcal{P H}$}

In this section, we will consider a subclass of polyharmonic functions:

$$
\begin{aligned}
\mathcal{P H}= & \left\{F: F=\sum_{n=0}^{p-1} r^{2 n} F_{n}, \text { where } F_{n}(z)^{\prime}\right. \text { 's are complex-valued harmonic } \\
& \text { functions in the unit disk } U\} .
\end{aligned}
$$

Some general properties of polyharmonic functions that are elements in $\mathcal{P H}$ are obtained, most importantly, a complex operator that preserves polyharmonicity is also introduced.

First, define the linear operator $\mathcal{L}$ by $\mathcal{L}=z \frac{\partial}{\partial z}-\bar{z} \frac{\partial}{\partial \bar{z}}$. The definition leads to the following two properties:

- $\mathcal{L}[\alpha f+\beta g]=\alpha \mathcal{L}[f]+\beta \mathcal{L}[g]$

- $\mathcal{L}[f g]=f \mathcal{L}[g]+g \mathcal{L}[f]$,

where $f, g$ are $C^{1}$ functions and $\alpha, \beta$ are complex constants.

Theorem 1. Let $F \in \mathcal{P H}$, where $F=\sum_{n=0}^{p-1} r^{2 n} F_{n}$. Then
a. $\mathcal{L}\left[r^{2 n}\right]=0$ for $n \geq 0$,
b. $\mathcal{L}[F]=\sum_{n=0}^{p-1} r^{2 n} \mathcal{L}\left[F_{n}\right]$,
c. $\mathcal{L}^{m}[F]=\sum_{n=0}^{p-1} r^{2 n} \mathcal{L}^{m}\left[F_{n}\right]$,

where $m \geq 2$ is an integer.

Proof. Let $F=\sum_{n=0}^{p-1} r^{2 n} F_{n} \in \mathcal{P} \mathcal{H}$.

a. The case $n=0$ is trivial. For $n \geq 1$ we have

$$
\begin{aligned}
\mathcal{L}\left[r^{2 n}\right] & =\mathcal{L}\left[\left(r^{2}\right)^{n}\right]=z\left[\left(r^{2}\right)^{n}\right]_{z}-\bar{z}\left[\left(r^{2}\right)^{n}\right]_{\bar{z}} \\
& =z\left[n r^{2 n-2} \bar{z}\right]-\bar{z}\left[n r^{2 n-2} z\right]=n r^{2 n}-n r^{2 n}=0 .
\end{aligned}
$$

b. Using the result in part (a) and the product rule property of the operator $\mathcal{L}$, we get

$$
\mathcal{L}[F]=\mathcal{L}\left[\sum_{n=0}^{p-1} r^{2 n} F_{n}\right]=\sum_{n=0}^{p-1} \mathcal{L}\left[r^{2 n}\right] F_{n}+\sum_{n=0}^{p-1} r^{2 n} \mathcal{L}\left[F_{n}\right]=\sum_{n=0}^{p-1} r^{2 n} \mathcal{L}\left[F_{n}\right]
$$


c. Using part (a) and (b) repeatedly (namely, mathematical induction), part (c) follows.

Corollary 1. The operator $\mathcal{L}$ is a $p$-harmonicity preserving operator.

Proof. This obviously follows from part (b) of Theorem 1 and the fact that $\mathcal{L}\left[F_{n}\right]$ is harmonic since $F_{n}$ 's are harmonic; it was proven in Lemma 3 (a) reference [2] that $\mathcal{L}$ is a harmonicity preserving operator. To preserve selfsufficiency one can easily prove that $\Delta \mathcal{L}=\mathcal{L} \Delta$. Then

$$
\Delta^{p} F=0 \quad \Rightarrow \quad \Delta^{p} \mathcal{L} F=\mathcal{L} \Delta^{p} F=0 \quad \Rightarrow \quad \mathcal{L} F \text { is polyharmonic. }
$$

Theorem 2. If $F$ is polyharmonic of order $p$, then $F_{z}$ and $F_{\bar{z}}$ are polyharmonic of order at most $p$.

Proof. Let $F=\sum_{n=0}^{p-1} r^{2 n} F_{n} \in \mathcal{P H}$, where the $F_{n}$ 's are harmonic. Then,

$$
\begin{aligned}
& F_{z}=\sum_{n=0}^{p-1}\left[n\left(r^{2}\right)^{n-1} \bar{z} F_{n}+\left(r^{2}\right)^{n}\left(F_{n}\right)_{z}\right]=\sum_{n=0}^{p-1}\left[n r^{2 n-2} \bar{z} F_{n}+r^{2 n}\left(F_{n}\right)_{z}\right], \\
& F_{\bar{z}}=\sum_{n=0}^{p-1}\left[n\left(r^{2}\right)^{n-1} z F_{n}+\left(r^{2}\right)^{n}\left(F_{n}\right)_{\bar{z}}\right]=\sum_{n=0}^{p-1}\left[n r^{2 n-2} z F_{n}+r^{2 n}\left(F_{n}\right)_{\bar{z}}\right] .
\end{aligned}
$$

Since $F_{n}$ is harmonic, that is $\left(F_{n}\right)_{z \bar{z}}=0$, therefore we have

$$
\left(\bar{z} F_{n}\right)_{z \bar{z}}=\left(\bar{z}\left(F_{n}\right)_{z}\right)_{\bar{z}}=\bar{z}\left(F_{n}\right)_{z \bar{z}}+\left(F_{n}\right)_{z}=\left(F_{n}\right)_{z}
$$

and hence

$$
\left(\bar{z} F_{n}\right)_{z z \overline{z z}}=\left(\left(F_{n}\right)_{z}\right)_{z \bar{z}}=\left(\left(F_{n}\right)_{z \bar{z}}\right)_{z}=0 .
$$

This means that $\bar{z} F_{n}$ is biharmonic and therefore it can be expressed in the form

$$
\bar{z} F_{n}=r^{2} K_{n}+G_{n},
$$

where $F_{n}$ and $G_{n}$ are harmonic. Substituting the latter expression into equation (2.1) yields

$$
\begin{aligned}
F_{z} & =\sum_{n=0}^{p-1}\left[n r^{2 n} K_{n}+n r^{2 n-2} G_{n}+r^{2 n}\left(F_{n}\right)_{z}\right] \\
& =\sum_{n=0}^{p-1} r^{2 n}\left(n K_{n}+\left(F_{n}\right)_{z}\right)+\sum_{n=0}^{p-1} r^{2 n-2} n G_{n} .
\end{aligned}
$$

Since $K_{n}, G_{n}$ and $F_{n}$ are harmonic so $n G_{n}, n K_{n},\left(F_{n}\right)_{z}$ and $\left(n K_{n}+\left(F_{n}\right)_{z}\right)$ are also harmonic. Therefore, the first term on the right-hand side of equation (2.3), namely, the term $\sum_{n=0}^{p-1} r^{2 n}\left(n K_{n}+\left(F_{n}\right)_{z}\right)$, is polyharmonic of order at most $p$ while the second term $\sum_{n=0}^{p-1} r^{2 n-2} n G_{n}$ is polyharmonic of order $p-1$. This means that the sum of both series, which is $F_{z}$, yields a polyharmonic function of order at most $p$. A similar argument can be used to show that $F_{\bar{z}}$ is polyharmonic of order at most $p$.

Alternatively, we can prove the theorem in the following way:

$$
\Delta^{p} F=0 \quad \Rightarrow \quad\left(\Delta^{p} F\right)_{z}=0 \quad \Rightarrow \quad \Delta^{p} F_{z}=0 .
$$




\section{Properties of the Subclass $\mathcal{C P H}$}

In this section, we will consider the following subclass of polyharmonic functions:

$$
\begin{aligned}
\mathcal{C P H}=\{ & G: G=\sum_{n=0}^{p-1} \lambda_{n} r^{2 n} F, \text { where } F(z) \text { is a complex-valued harmonic } \\
& \text { mapping in the unit disk } U \text { and } \lambda_{n}, n=0,1, \ldots, p-1 \\
& \left.\left(\lambda_{0}^{2}+\lambda_{1}^{2}+\cdots+\lambda_{p-1}^{2} \neq 0\right), \text { are constants }\right\} .
\end{aligned}
$$

The $\lambda_{i}$ 's are real constants and are not all zero. Though we need to emphasize that some of the theorems and results included in this paper can be easily generalized and are true for complex values of $\lambda_{i}$ 's. Some geometrical properties related to starlikeness, convexity and univalence for elements in $\mathcal{C P} \mathcal{H}$ are obtained. Further, we extend Landau's theorem to functions belonging to this subclass, and show that the Goodman-Saff conjecture is true for mappings belonging to $\mathcal{C P} \mathcal{H}$.

Theorem 3. Let $G=\sum_{n=0}^{p-1} \lambda_{n} r^{2 n} F \in \mathcal{C P} \mathcal{H}$. Then

$$
\text { a) } \frac{\mathcal{L}[G]}{G}=\frac{\mathcal{L}[F]}{F}, \quad \text { b) } \frac{\mathcal{L}^{m}[G]}{G}=\frac{\mathcal{L}^{m}[F]}{F}, \quad \text { c) } \frac{\mathcal{L}^{m}[G]}{\mathcal{L}[G]}=\frac{\mathcal{L}^{m}[F]}{\mathcal{L}[F]},
$$

where $m \geq 2$ is an integer.

Proof. From part (b) of Theorem 1 we have $\mathcal{L}[G]=\sum_{n=0}^{p-1} \lambda_{n} r^{2 n} \mathcal{L}[F]$. Dividing both sides by $G$, part (a) follows. The following result can be obtained by mathematical induction:

$$
\mathcal{L}^{m}[G]=\sum_{n=0}^{p-1} \lambda_{n} r^{2 n} \mathcal{L}^{m}[F],
$$

hence part (b) follows. The proof of part (c) is similar to part (b).

Theorem 4. Let $G=\sum_{n=0}^{p-1} \lambda_{n} r^{2 n} F \in \mathcal{C P H}$ be univalent, where $F$ is harmonic and $F(0)=0$. Then

a. $G$ is starlike if $F$ is starlike.

b. Assume $\mathcal{L}[G] \neq 0$ and $\mathcal{L}[F] \neq 0$ for $z \neq 0$. Then $G$ is convex if $F$ is convex.

c. If $F$ is convex and $\mathcal{L}[G]$ is univalent then $\mathcal{L}[G]$ is starlike.

Proof. From the definition of the operator $\mathcal{L}$ and that for starlikeness, $F$ is starlike if and only if

$$
\operatorname{Re}\left(\frac{z F_{z}-\bar{z} F_{\bar{z}}}{F}\right)=\operatorname{Re}\left(\frac{\mathcal{L}[F]}{F}\right)>0 .
$$


From Theorem 3.a and the fact that $F$ is starlike implies that

$$
\operatorname{Re}\left(\frac{\mathcal{L}[G]}{G}\right)=\operatorname{Re}\left(\frac{\mathcal{L}[F]}{F}\right)>0 .
$$

This proves part (a). We have

$$
\frac{\partial}{\partial t} F\left(r e^{i t}\right)=F_{z} \frac{\partial z}{\partial t}+F_{\bar{z}} \frac{\partial \bar{z}}{\partial t}=i z F_{z}-i \bar{z} F_{\bar{z}}=i \mathcal{L}[F] .
$$

Therefore

$$
\begin{aligned}
\frac{\partial}{\partial t}\left(\arg \frac{\partial F\left(r e^{i t}\right)}{\partial t}\right) & =\operatorname{Im}\left(\frac{\frac{\partial^{2} F\left(r e^{i t}\right)}{\partial t^{2}}}{\frac{\partial}{\partial t} F\left(r e^{i t}\right)}\right)=\operatorname{Im}\left(\frac{\frac{\partial}{\partial t} \mathcal{L}[F]}{\mathcal{L}[F]}\right)=\operatorname{Im}\left(\frac{\mathcal{L}\left[\frac{\partial}{\partial t} F\right]}{\mathcal{L}[F]}\right) \\
& =\operatorname{Im} \frac{\mathcal{L}[i \mathcal{L}[F]]}{\mathcal{L}[F]}=\operatorname{Re} \frac{\mathcal{L}^{2}[F]}{\mathcal{L}[F]}
\end{aligned}
$$

Alternatively we have

$$
\frac{\partial}{\partial t}\left(\arg \frac{\partial F\left(r e^{i t}\right)}{\partial t}\right)=\operatorname{Re} \frac{\mathcal{L}[i \mathcal{L}[F]]}{i \mathcal{L}[F]}=\operatorname{Re} \frac{\mathcal{L}^{2}[F]}{\mathcal{L}[F]} .
$$

From the definition of convexity and the latter equation it follows that $F$ is convex if and only if $\operatorname{Re} \frac{\mathcal{L}^{2}[F]}{\mathcal{L}[F]}>0$ provided $\mathcal{L}[F] \neq 0$ for $z \neq 0$. From Theorem 3.c and the fact that $F$ is convex we conclude that

$$
\operatorname{Re}\left(\frac{\mathcal{L}^{2}[G]}{\mathcal{L}[G]}\right)=\operatorname{Re}\left(\frac{\mathcal{L}^{2}[F]}{\mathcal{L}[F]}\right)>0
$$

This means that $G$ is convex and so the proof of part (b) is complete.

$F$ is convex implies that $\operatorname{Re}\left(\frac{\mathcal{L}^{2}[F]}{\mathcal{L}[F]}\right)>0$. Therefore from Theorem 3.c we have $\operatorname{Re}\left(\frac{\mathcal{L}^{2}[G]}{\mathcal{L}[G]}\right)>0$ as well. This means that $\mathcal{L}[G]$ is starlike and the proof is complete.

Theorem 5. Let $G=\sum_{n=0}^{p-1} \lambda_{n} r^{2 n} F \in \mathcal{C P H}$ where $\lambda_{n}$ 's are real. Then the Jacobian of $G$, denoted by $J_{G}$, is given by

$$
J_{G}=\left(\sum_{n=0}^{p-1} \lambda_{n} r^{2 n}\right)^{2} J_{F}+2\left(\sum_{n=0}^{p-1} n \lambda_{n} r^{2 n-2}\right)\left(\sum_{n=0}^{p-1} \lambda_{n} r^{2 n}\right)|F|^{2} F_{s t},
$$

where $F_{s t}=\operatorname{Re}\left(\frac{z F_{z}-\bar{z} F_{\bar{z}}}{F}\right)$ denotes the measure of starlikeness.

Proof. Let $G=\sum_{n=0}^{p-1} \lambda_{n} r^{2 n} F \in \mathcal{C P H}$. Then

$$
G_{z}=\sum_{n=0}^{p-1}\left[n \lambda_{n} r^{2 n-2} \bar{z} F+\lambda_{n} r^{2 n} F_{z}\right], \quad G_{\bar{z}}=\sum_{n=0}^{p-1}\left[n \lambda_{n} r^{2 n-2} z F+\lambda_{n} r^{2 n} F_{\bar{z}}\right] .
$$


Hence we have

$$
\begin{aligned}
\left|G_{z}\right|^{2}= & \left(\sum_{n=0}^{p-1}\left[n \lambda_{n} r^{2 n-2} \bar{z} F+\lambda_{n} r^{2 n} F_{z}\right]\right)\left(\sum_{n=0}^{p-1}\left[n \lambda_{n} r^{2 n-2} z \bar{F}+\lambda_{n} r^{2 n} \overline{F_{z}}\right]\right) \\
= & \left(\sum_{n=0}^{p-1} n \lambda_{n} r^{2 n-1}\right)^{2}|F|^{2}+\left(\sum_{n=0}^{p-1} n \lambda_{n} r^{2 n-2}\right)\left(\sum_{n=0}^{p-1} \lambda_{n} r^{2 n}\right) \overline{z F} \overline{F_{z}} \\
& +\left(\sum_{n=0}^{p-1} \lambda_{n} r^{2 n}\right)\left(\sum_{n=0}^{p-1} n \lambda_{n} r^{2 n-2}\right) z \bar{F} F_{z}+\left(\sum_{n=0}^{p-1} \lambda_{n} r^{2 n}\right)^{2}\left|F_{z}\right|^{2} \\
= & \left(\sum_{n=0}^{p-1} n \lambda_{n} r^{2 n-1}\right)^{2}|F|^{2}+2\left(\sum_{n=0}^{p-1} n \lambda_{n} r^{2 n-2}\right)\left(\sum_{n=0}^{p-1} \lambda_{n} r^{2 n}\right) \operatorname{Re}\left(z \bar{F} F_{z}\right) \\
& +\left(\sum_{n=0}^{p-1} \lambda_{n} r^{2 n}\right)^{2}\left|F_{z}\right|^{2} .
\end{aligned}
$$

The equalities in the previous equations as well as the subsequent ones are true for real $\lambda_{n}$ 's only. Similarly

$$
\begin{aligned}
\left|G_{\bar{z}}\right|^{2}= & \left(\sum_{n=0}^{p-1}\left[n \lambda_{n} r^{2 n-2} z F+\lambda_{n} r^{2 n} F_{\bar{z}}\right]\right)\left(\sum_{n=0}^{p-1}\left[n \lambda_{n} r^{2 n-2} \bar{z} \bar{F}+\lambda_{n} r^{2 n} \overline{F_{\bar{z}}}\right]\right) \\
= & \left(\sum_{n=0}^{p-1} n \lambda_{n} r^{2 n-1}\right)^{2}|F|^{2}+2\left(\sum_{n=0}^{p-1} n \lambda_{n} r^{2 n-2}\right)\left(\sum_{n=0}^{p-1} \lambda_{n} r^{2 n}\right) \operatorname{Re}\left(\bar{z} \bar{F} F_{\bar{z}}\right) \\
& +\left(\sum_{n=0}^{p-1} \lambda_{n} r^{2 n}\right)^{2}\left|F_{\bar{z}}\right|^{2} .
\end{aligned}
$$

Therefore

$$
\begin{aligned}
J_{G}= & \left|G_{z}\right|^{2}-\left|G_{\bar{z}}\right|^{2}=2\left(\sum_{n=0}^{p-1} n \lambda_{n} r^{2 n-2}\right)\left(\sum_{n=0}^{p-1} \lambda_{n} r^{2 n}\right) \operatorname{Re}\left(z \bar{F} F_{z}-\bar{z} \bar{F} F_{\bar{z}}\right) \\
& +\left(\sum_{n=0}^{p-1} \lambda_{n} r^{2 n}\right)^{2}\left(\left|F_{z}\right|^{2}-\left|F_{\bar{z}}\right|^{2}\right) \\
= & 2\left(\sum_{n=0}^{p-1} n \lambda_{n} r^{2 n-2}\right)\left(\sum_{n=0}^{p-1} \lambda_{n} r^{2 n}\right)|F|^{2} F_{s t}+\left(\sum_{n=0}^{p-1} \lambda_{n} r^{2 n}\right)^{2} J_{F} \cdot
\end{aligned}
$$

Corollary 2. Let $G=\sum_{n=0}^{p-1} \lambda_{n} r^{2 n} F \in \mathcal{C P} \mathcal{H}$. Assume the function $F$ is starlike, orientation preserving and $\lambda_{n}>0, n=0,1, \ldots, p-1$, then $G$ is locally univalent in $U$.

Proof. $F$ is starlike implies that $F_{\text {st }}>0$. Further we have $J_{F}>0$ which follows from the fact that $F$ is orientation preserving. Since it is assumed that $\lambda_{n}>0, n=0,1, \ldots, p-1$, it follows from Theorem 5 that $J_{G}(z)>0$, that is $G$ is orientation preserving and hence $J_{G}(z) \neq 0$ which means that $G$ is locally univalent. 
Theorem 6. Let $G=\sum_{n=0}^{p-1} \lambda_{n} r^{2 n} F \in \mathcal{C P H}$. Then
a. $\quad-i \frac{\partial G\left(r e^{i t}\right)}{\partial t}=\sum_{n=0}^{p-1} \lambda_{n} r^{2 n}\left[z F_{z}-\bar{z} F_{\bar{z}}\right]$
b. $-\frac{\partial^{2} G\left(r e^{i t}\right)}{\partial t^{2}}=\sum_{n=0}^{p-1} \lambda_{n} r^{2 n}\left[z F_{z}+\bar{z} F_{\bar{z}}+z^{2} F_{z z}+\bar{z}^{2} F_{\overline{z z}}\right]$.

Proof. We have

$$
\begin{array}{cc}
G_{z}=\sum_{n=0}^{p-1}\left[\lambda_{n} r^{2 n} F_{z}+n \lambda_{n} r^{2 n-2} \bar{z} F\right], & G_{\bar{z}}=\sum_{n=0}^{p-1}\left[\lambda_{n} r^{2 n} F_{\bar{z}}+n \lambda_{n} r^{2 n-2} z F\right], \\
z G_{z}=\sum_{n=0}^{p-1} \lambda_{n} r^{2 n}\left[z F_{z}+n F\right], & \bar{z} G_{\bar{z}}=\sum_{n=0}^{p-1} \lambda_{n} r^{2 n}\left[\bar{z} F_{\bar{z}}+n F\right] .
\end{array}
$$

Applying the chain rule and manipulating the previous equations yields

$$
\begin{aligned}
\frac{\partial G\left(r e^{i t}\right)}{\partial t} & =i z G_{z}-i \bar{z} G_{\bar{z}}=i\left(z G_{z}-\bar{z} G_{\bar{z}}\right) \\
& =i \sum_{n=0}^{p-1} \lambda_{n} r^{2 n}\left[z F_{z}+n F\right]-i \sum_{n=0}^{p-1} \lambda_{n} r^{2 n}\left[\bar{z} F_{\bar{z}}+n F\right] \\
& =i \sum_{n=0}^{p-1} \lambda_{n} r^{2 n}\left[z F_{z}-\bar{z} F_{\bar{z}}\right]
\end{aligned}
$$

and therefore part (a) follows. This result is a consequence of Theorem 3.a and can be proved as follows:

$$
-i \frac{\partial G\left(r e^{i t}\right)}{\partial t}=\mathcal{L}[G]=\mathcal{L}[F] G / F=\sum_{n=0}^{p-1} \lambda_{n} r^{2 n} \mathcal{L}[F] .
$$

Further calculation and noting that $F$ is harmonic, that is $F_{z \bar{z}}=0$, it follows that

$$
\begin{aligned}
G_{z \bar{z}}= & \sum_{n=0}^{p-1}\left[n \lambda_{n} r^{2 n-2} z F_{z}+n(n-1) \lambda_{n} r^{2 n-4} z \bar{z} F\right. \\
& \left.+n \lambda_{n} r^{2 n-2} F+n \lambda_{n} r^{2 n-2} \bar{z} F_{\bar{z}}\right], \\
2 r^{2} G_{z \bar{z}}= & \sum_{n=0}^{p-1} 2 n \lambda_{n} r^{2 n}\left[z F_{z}+\bar{z} F_{\bar{z}}+n F\right] . \\
G_{z z}= & \sum_{n=0}^{p-1}\left[n \lambda_{n} r^{2 n-2} \bar{z} F_{z}+\lambda_{n} r^{2 n} F_{z z}+n(n-1) \lambda_{n} r^{2 n-4} \bar{z}^{2} F+n \lambda_{n} r^{2 n-2} \bar{z} F_{z}\right], \\
z^{2} G_{z z}= & \sum_{n=0}^{p-1} \lambda_{n} r^{2 n}\left[2 n z F_{z}+z^{2} F_{z z}+n(n-1) F\right],
\end{aligned}
$$


and

$$
\begin{aligned}
G_{\overline{z z}} & =\sum_{n=0}^{p-1}\left[n \lambda_{n} r^{2 n-2} z F_{\bar{z}}+\lambda_{n} r^{2 n} F_{\overline{z z}}+n(n-1) \lambda_{n} r^{2 n-4} z^{2} F+n \lambda_{n} r^{2 n-2} z F_{z}\right], \\
\bar{z}^{2} G_{\overline{z z}} & =\sum_{n=0}^{p-1} \lambda_{n} r^{2 n-2}\left[2 n \bar{z} F_{\bar{z}}+\bar{z}^{2} F_{\overline{z z}}+n(n-1) F\right] .
\end{aligned}
$$

Again, using the chain rule and the above equations, we have

$$
\begin{aligned}
\frac{\partial^{2} G\left(r e^{i t}\right)}{\partial t^{2}}= & \frac{\partial}{\partial t}\left[i z G_{z}-i \bar{z} G_{\bar{z}}\right]=\frac{\partial}{\partial z}\left[i z G_{z}-i \bar{z} G_{\bar{z}}\right] \frac{\partial z}{\partial t}+\frac{\partial}{\partial \bar{z}}\left[i z G_{z}-i \bar{z} G_{\bar{z}}\right] \frac{\partial \bar{z}}{\partial t} \\
= & -z G_{z}-\bar{z} G_{\bar{z}}+2|z|^{2} G_{z \bar{z}}-z^{2} G_{z z}-\bar{z}^{2} G_{\overline{z z}} \\
= & -\sum_{n=0}^{p-1} \lambda_{n} r^{2 n}\left[\left(z F_{z}+n F\right)+\left(\bar{z} F_{\bar{z}}+n F\right)-2 n\left(z F_{z}+\bar{z} F_{\bar{z}}+n F\right)\right. \\
& \left.+\left(2 n z F_{z}+z^{2} F_{z z}+n(n-1) F\right)+\left(2 n \bar{z} F_{\bar{z}}+\bar{z}^{2} F_{\overline{z z}}+n(n-1) F\right)\right] \\
= & -\sum_{n=0}^{p-1} \lambda_{n} r^{2 n}\left[z F_{z}+\bar{z} F_{\bar{z}}-2 n z F_{z}-2 n \bar{z} F_{\bar{z}}+2 n z F_{z}+z^{2} F_{z z}\right. \\
& \left.+2 n \bar{z} F_{\bar{z}}+\bar{z}^{2} F_{\overline{z z}}\right] \\
& p-1 \\
& \sum_{n=0} \lambda_{n} r^{2 n}\left[z F_{z}+\bar{z} F_{\bar{z}}+z^{2} F_{z z}+\bar{z}^{2} F_{\overline{z z}}\right] .
\end{aligned}
$$

Consequently, the proof of part (b) is complete. This result can be proven in a different fashion with the help of Theorem 3.b as follows:

$$
-\frac{\partial^{2} G\left(r e^{i t}\right)}{\partial t^{2}}=\mathcal{L}^{2}[G]=\mathcal{L}^{2}[F] G / F=\left(z F_{z}+\bar{z} F_{\bar{z}}+z^{2} F_{z z}+\bar{z}^{2} F_{\overline{z z}}\right) G / F .
$$

Corollary 3. Let $G=\sum_{n=0}^{p-1} \lambda_{n} r^{2 n} F \in \mathcal{C P H}$. Then

$$
\frac{\partial}{\partial t}\left(\arg \frac{\partial G\left(r e^{i t}\right)}{\partial t}\right)=\frac{\partial}{\partial t}\left(\arg \frac{\partial F\left(r e^{i t}\right)}{\partial t}\right) .
$$

Proof. Using Theorem 6 yields

$$
\begin{aligned}
& \frac{\partial}{\partial t}\left(\arg \frac{\partial G\left(r e^{i t}\right)}{\partial t}\right) \operatorname{Im}\left(\frac{\frac{\partial^{2} G\left(r e^{i t}\right)}{\partial t^{2}}}{\frac{\partial G\left(r e^{i t}\right)}{\partial t}}\right) \\
& =\operatorname{Im}\left(\frac{-\sum_{n=0}^{p-1} \lambda_{n} r^{2 n}\left[z F_{z}+\bar{z} F_{\bar{z}}+z^{2} F_{z z}+\bar{z}^{2} F_{\overline{z z}}\right]}{i \sum_{n=0}^{p-1} \lambda_{n} r^{2 n}\left[z F_{z}-\bar{z} F_{\bar{z}}\right]}\right) \\
& =\operatorname{Re}\left(\frac{z F_{z}+\bar{z} F_{\bar{z}}+z^{2} F_{z z}+\bar{z}^{2} F_{\overline{z z}}}{z F_{z}-\bar{z} F_{\bar{z}}}\right)=\operatorname{Im}\left(\frac{\frac{\partial^{2} F\left(r e^{i t}\right)}{\partial t^{2}}}{\frac{\partial F\left(r e^{i t}\right)}{\partial t}}\right)=\frac{\partial}{\partial t}\left(\arg \frac{\partial F\left(r e^{i t}\right)}{\partial t}\right) \cdot \square
\end{aligned}
$$

The Goodman-Saff conjecture is valid for functions belonging to the class $\mathcal{C P H}$. This is the statement of the next theorem. 
Theorem 7. Any non-constant complex-valued function $G \in \mathcal{C P H}$ sends the subdisk $|z|<r$ onto a convex region for $r \leq \sqrt{2}-1$.

Proof. Let $G=\sum_{n=0}^{p-1} \lambda_{n} r^{2 n} F \in \mathcal{C P H}$, where $F$ is harmonic. We need the following theorem by Ruscheweyh and Salinas [18] (see Theorem 1), namely the Goodman-Saff conjecture for harmonic functions:

Let $K_{H}$ denote the class of all complex-valued harmonic univalent functions $f$ on the unit disk $U$ with $f(U)$ convex in the direction $e^{i \phi}$. If $f \in K_{H}(\phi)$, $0<r \leq r_{0}=\sqrt{2}-1$, then $f(r z) \in K_{H}(\phi)$.

Now we can prove the result: since $G$ being convex, it follows from the definition of convexity that

$$
G \text { is convex } \Longleftrightarrow \frac{\partial}{\partial t}\left(\arg \frac{\partial G\left(r e^{i t}\right)}{\partial t}\right)>0 .
$$

Since $F$ is harmonic and if we further assume that it is convex in $0<r \leq r_{0}=$ $\sqrt{2}-1$, then Corollary 3 implies that $G$ is also convex because

$$
\frac{\partial}{\partial t}\left(\arg \frac{\partial G\left(r e^{i t}\right)}{\partial t}\right)=\frac{\partial}{\partial t}\left(\arg \frac{\partial F\left(r e^{i t}\right)}{\partial t}\right)>0 .
$$

Thus the conclusion of the theorem follows.

In the subsequent theorem, we show that Landau's theorem extends to bounded polyharmonic mappings on the unit disk belonging to the class $\mathcal{C P} \mathcal{H}$.

Theorem 8. Let $G=\sum_{n=0}^{p-1} \lambda_{n} r^{2 n} F \in \mathcal{C P H}, z=r e^{i \theta}$, be a p-harmonic mapping of the unit disk $U$, where $F$ is harmonic, such that $F(0)=0, \lambda_{0} \neq 0$, $J_{G}(0)=1$ and $|F|$ is bounded by $M$. Then there is a constant $0<\rho_{1}<1$ so that $G$ is univalent in $|z|<\rho_{1}$, where $\rho_{1}$ is the unique solution of the equation

$$
\begin{aligned}
& \frac{\pi}{4\left|\lambda_{0}\right| M}-2 \rho_{1} M \sum_{n=1}^{p-1} n\left|\lambda_{n}\right| \rho_{1}^{2 n-2}-\frac{2 M \rho_{1}^{2}}{\left(1-\rho_{1}\right)^{2}} \sum_{n=1}^{p-1}\left|\lambda_{n}\right| \rho_{1}^{2 n} \\
& -2 M\left|\lambda_{0}\right|\left(\frac{1}{\left(1-\rho_{1}\right)^{2}}-1\right)=0
\end{aligned}
$$

and $G\left(U_{\rho_{1}}\right)$ contains a disk $U_{R_{1}}$ where

$$
R_{1}=\frac{\pi}{4\left|\lambda_{0}\right| M} \rho_{1}-\frac{2 M \rho_{1}}{1-\rho_{1}}\left(\sum_{n=1}^{p-1}\left|\lambda_{n}\right| \rho_{1}^{2 n}+\left|\lambda_{0}\right| \rho_{1}\right) .
$$

Proof. Let $G \in \mathcal{C P} \mathcal{H}$. We have

$$
G=\sum_{n=0}^{p-1} \lambda_{n} r^{2 n} F=\sum_{n=1}^{p-1} \lambda_{n} r^{2 n} F+\lambda_{0} F
$$


where $F$ is harmonic, so that $F=f_{1}(z)+\overline{f_{2}(z)}$ where $f_{1}$ and $f_{2}$ are analytic in $D$. Therefore

$$
\begin{aligned}
G_{z} & =\sum_{n=1}^{p-1}\left[n \lambda_{n} r^{2 n-2} \bar{z} F+\lambda_{n} r^{2 n} F_{z}\right]+\lambda_{0} F_{z}, \\
G_{\bar{z}} & =\sum_{n=1}^{p-1}\left[n \lambda_{n} r^{2 n-2} z F+\lambda_{n} r^{2 n} F_{\bar{z}}\right]+\lambda_{0} F_{\bar{z}} .
\end{aligned}
$$

Let $F(z)=\sum_{0}^{\infty} a_{n} z^{n}+\overline{\sum_{0}^{\infty} b_{n} z^{n}}$. For fixed $0<\rho<1$, choose $z_{1}$, $z_{2}$ with $z_{1} \neq z_{2},\left|z_{1}\right|<\rho$ and $\left|z_{2}\right|<\rho$. Then

$$
\begin{aligned}
G\left(z_{1}\right)-G\left(z_{2}\right)= & \int_{\left[z_{1}, z_{2}\right]} G_{z}(z) d z+G_{\bar{z}}(z) d \bar{z} \\
= & \int_{\left[z_{1}, z_{2}\right]}\left[\left(\sum_{n=1}^{p-1} n \lambda_{n} r^{2 n-2} \bar{z} F+\sum_{n=1}^{p-1} \lambda_{n} r^{2 n} f_{1}^{\prime}+\lambda_{0} F_{z}\right) d z\right. \\
& \left.+\left(\sum_{n=1}^{p-1} n \lambda_{n} r^{2 n-2} z F+\sum_{n=1}^{p-1} \lambda_{n} r^{2 n} f_{2}^{\prime}+\lambda_{0} \overline{f_{2}^{\prime}}\right) d \bar{z}\right]
\end{aligned}
$$

where $\left[z_{1}, z_{2}\right]$ is the line-segment joining $z_{1}$ with $z_{2}$. We have

$$
J_{F}=\left|F_{z}\right|^{2}-\left|F_{\bar{z}}\right|^{2}=\left(\left|F_{z}\right|-\left|F_{\bar{z}}\right|\right)\left(\left|F_{z}\right|+\left|F_{\bar{z}}\right|\right)=\lambda_{F} \cdot \Lambda_{F},
$$

where $\lambda_{F}=\left|F_{z}\right|-\left|F_{\bar{z}}\right|$ and $\Lambda_{F}=\left|F_{z}\right|+\left|F_{\bar{z}}\right|$. Note that

$$
1=J_{G}(0)=\left|G_{z}(0)\right|^{2}-\left|G_{\bar{z}}(0)\right|^{2}=\left(\left|F_{z}(0)\right|^{2}-\left|F_{\bar{z}}(0)\right|^{2}\right)\left|\lambda_{0}\right|^{2}=\left|\lambda_{0}\right|^{2} J_{F}(0) .
$$

Thus

$$
\lambda_{F}(0)=\frac{J_{F}(0)}{\Lambda_{F}(0)}=\frac{J_{G}(0)}{\left|\lambda_{0}\right|^{2} \Lambda_{F}(0)}=\frac{1}{\left|\lambda_{0}\right|^{2} \Lambda_{F}(0)} .
$$

It follows by Schwarz lemma (see Lemma 1 in reference [1]) that

$$
\lambda_{F}(0)=\frac{1}{\left|\lambda_{0}\right|^{2} \Lambda_{F}(0)} \geq \frac{\pi}{4\left|\lambda_{0}\right|^{2} M} .
$$

Taking advantage of the triangle inequality

$$
\begin{aligned}
\left|\int\left(f_{1}(z)+f_{2}(z)\right) d z\right| & \geq\left|\int\right| f_{1}(z)\left|d z-\int\right| f_{2}(z)|d z| \\
& \geq \int\left|f_{1}(z)\right| d z-\int\left|f_{2}(z)\right| d z
\end{aligned}
$$

we have

$$
\begin{aligned}
\left|\int_{\left[z_{1}, z_{2}\right]} \lambda_{0}\left(F_{z}(0) d z+F_{\bar{z}}(0) d \bar{z}\right)\right| & \geq \int_{\left[z_{1}, z_{2}\right]}\left|\lambda_{0} F_{z}(0) d z\right|-\int_{\left[z_{1}, z_{2}\right]}\left|\lambda_{0} F_{\bar{z}}(0) d \bar{z}\right| \\
& =\left|\lambda_{0}\right| \int_{\left[z_{1}, z_{2}\right]}\left|F_{z}(0)\right||d z|-\left|\lambda_{0}\right| \int_{\left[z_{1}, z_{2}\right]}\left|F_{\bar{z}}(0)\right||d \bar{z}| .
\end{aligned}
$$


Since $|d z|=|d \bar{z}|$, we have

$$
\begin{aligned}
& \left|\int_{\left[z_{1}, z_{2}\right]} \lambda_{0}\left(F_{z}(0) d z+F_{\bar{z}}(0) d \bar{z}\right)\right| \geq\left|\lambda_{0}\right|\left(\int_{\left[z_{1}, z_{2}\right]}\left|F_{z}(0)\right||d z|-\int_{\left[z_{1}, z_{2}\right]}\left|F_{\bar{z}}(0)\right||d z|\right) \\
& \quad=\left|\lambda_{0}\right|\left(\int_{\left[z_{1}, z_{2}\right]}\left|F_{z}(0)\right|-\left|F_{\bar{z}}(0)\right||d z|\right)=\left|\lambda_{0}\right|\left(\int_{\left[z_{1}, z_{2}\right]} \lambda_{F}(0)|d z|\right) \\
& \quad=\left|z_{2}-z_{1}\right|\left|\lambda_{0}\right| \lambda_{F}(0) .
\end{aligned}
$$

Therefore

$$
\begin{aligned}
& \left|G\left(z_{1}\right)-G\left(z_{2}\right)\right| \geq\left|\int_{\left[z_{1}, z_{2}\right]} \lambda_{0}\left(F_{z}(0) d z+F_{\bar{z}}(0) d \bar{z}\right)\right| \\
& -\mid \int_{\left[z_{1}, z_{2}\right]} \sum_{n=1}^{p-1} n \lambda_{n} r^{2 n-2} F(z)(\bar{z} d z+z d \bar{z})+\int \sum_{\left[z_{1}, z_{2}\right]} \sum_{n=1}^{p-1} \lambda_{n} r^{2 n}\left(f_{1}^{\prime}(z) d z+\overline{f_{2}^{\prime}(z)} d \bar{z}\right) \\
& +\int_{\left[z_{1}, z_{2}\right]} \lambda_{0}\left(F_{z}(z)-F_{z}(0)\right) d z+\lambda_{0}\left(F_{\bar{z}}(z)-F_{\bar{z}}(0)\right) d \bar{z} \mid \\
& \geq\left|z_{2}-z_{1}\right|\left(\left|\lambda_{0}\right| \lambda_{F}(0)-2 \rho M \sum_{n=1}^{p-1} n\left|\lambda_{n}\right| \rho^{2 n-2}\right. \\
& \left.-\sum_{n=1}^{p-1}\left|\lambda_{n}\right| \rho^{2 n} \sum_{n=1}^{\infty}\left(\left|a_{n}\right|+\left|b_{n}\right|\right) n \rho^{n-1}-\sum_{n=2}^{\infty}\left(\left|a_{n}\right|+\left|b_{n}\right|\right) n\left|\lambda_{0}\right| \rho^{n-1}\right) \\
& \geq\left|z_{2}-z_{1}\right|\left(\left|\lambda_{0}\right| \lambda_{F}(0)-2 \rho M \sum_{n=1}^{p-1} n\left|\lambda_{n}\right| \rho^{2 n-2}\right. \\
& \left.-2 M \sum_{n=1}^{p-1}\left|\lambda_{n}\right| \rho^{2 n} \sum_{n=1}^{\infty} n \rho^{n-1}-2 M\left|\lambda_{0}\right| \sum_{n=2}^{\infty} n \rho^{n-1}\right) \\
& \geq\left|z_{2}-z_{1}\right|\left(\frac{\pi}{4\left|\lambda_{0}\right| M}-2 \rho M \sum_{n=1}^{p-1} n\left|\lambda_{n}\right| \rho^{2 n-2}\right. \\
& \left.-\frac{2 M \rho^{2}}{(1-\rho)^{2}} \sum_{n=1}^{p-1}\left|\lambda_{n}\right| \rho^{2 n}-2 M\left|\lambda_{0}\right|\left(\frac{1}{(1-\rho)^{2}}-1\right)\right) \text {. }
\end{aligned}
$$

In the latter inequality, the function $\Lambda(\rho)$, defined by

$$
\begin{aligned}
\Lambda(\rho)= & \frac{\pi}{4\left|\lambda_{0}\right| M}-2 \rho M \sum_{n=1}^{p-1} n\left|\lambda_{n}\right| \rho^{2 n-2}-\frac{2 M \rho^{2}}{(1-\rho)^{2}} \sum_{n=1}^{p-1}\left|\lambda_{n}\right| \rho^{2 n} \\
& -2 M\left|\lambda_{0}\right|\left(\frac{1}{(1-\rho)^{2}}-1\right),
\end{aligned}
$$


can be easily shown to be a decreasing function of $\rho$ on the interval $(0,1)$. Further, we have $\lim _{\rho \rightarrow 0^{+}} \Lambda(\rho)=\frac{\pi}{4\left|\lambda_{0}\right| M}>0$ and $\lim _{\rho \rightarrow 1^{-}} \Lambda(\rho)=-\infty$. This implies that there is a unique root $\rho_{1} \in(0,1)$ of the function $\Lambda(\rho)$. This shows that $\left|G\left(z_{1}\right)-G\left(z_{2}\right)\right| \geq\left|z_{2}-z_{1}\right| \Lambda(\rho)>0$ for any two distinct points $z_{1}, z_{2} \in|z|<\rho_{1}$, which proves the univalency of $f$ in the disk $U_{\rho_{1}}$. Finally, proceeding in the same way, we consider any $z$ with $|z|=\rho_{1}$. Then, we have

$$
\begin{aligned}
|G(z)| \geq & \left|\lambda_{0}\right|\left|a_{1} z+b_{1} \bar{z}\right|-\sum_{n=1}^{p-1}\left|\lambda_{n}\right| \rho_{1}^{2 n}\left|\sum_{n=1}^{\infty}\left(a_{n} z^{n}+b_{n} \bar{z}^{n}\right)\right| \\
& -\left|\lambda_{0}\right|\left|\sum_{n=2}^{\infty}\left(a_{n} z^{n}+b_{n} \bar{z}^{n}\right)\right| \\
\geq & \frac{\pi}{4\left|\lambda_{0}\right| M} \rho_{1}-\sum_{n=1}^{p-1}\left|\lambda_{n}\right| \rho_{1}^{2 n} \frac{2 M \rho_{1}}{1-\rho_{1}}-\left|\lambda_{0}\right| \frac{2 M \rho_{1}^{2}}{1-\rho_{1}} \\
= & \frac{\pi}{4\left|\lambda_{0}\right| M} \rho_{1}-\frac{2 M \rho_{1}}{1-\rho_{1}}\left(\sum_{n=1}^{p-1}\left|\lambda_{n}\right| \rho_{1}^{2 n}+\left|\lambda_{0}\right| \rho_{1}\right) .
\end{aligned}
$$

The result related to Landau's Theorem 8 was proved for biharmonic function in reference [1], then Liu [16] improved the results in [1] and obtained better estimates by establishing better coefficient estimates for bounded and normalized planar harmonic mappings. In the next theorem, we will modify the result in Theorem 8 by adopting the method of the proof in [17] in which an improved result of Landau's theorem was proved for biharmonic mappings. It is worth mentioning that we will consider a slightly wider class of functions than those belonging to $\mathcal{C P H}$. We need first the following two lemmas (see [16]).

Lemma 1. [16] Suppose that $f(z)=h(z)+\overline{g(z)}$ is a harmonic mapping of the unit disk $\mathcal{U}$ where $h(z)=\sum_{n=1}^{\infty} a_{n} z^{n}$ and $g(z)=\sum_{n=1}^{\infty} b_{n} z^{n}$ are analytic on $\mathcal{U}$. If $|f(z)| \leq M$ for $z \in \mathcal{U}$, then

$$
\left|a_{n}\right|,\left|b_{n}\right| \leq M, \quad n=1,2, \ldots
$$

Each of the above inequalities is sharp, the extremal functions $f_{1}(z)=M z^{n}$ and $f_{2}(z)=M \bar{z}^{n}$ yield their equalities.

Lemma 2. [16] Suppose that $f(z)=h(z)+\overline{g(z)}$ is a harmonic mapping of the unit disk $\mathcal{U}$ with $h(z)=\sum_{n=1}^{\infty} a_{n} z^{n}$ and $g(z)=\sum_{n=1}^{\infty} b_{n} z^{n}$ for $z \in \mathcal{U}$. If $J_{f}(0)=1$ and $|f(z)|<M$, then

$$
\left|a_{n}\right|,\left|b_{n}\right| \leq \sqrt{M^{2}-1}, \quad n=2,3, \ldots,
$$

and

$$
\left|a_{n}\right|+\left|b_{n}\right| \leq \sqrt{2 M^{2}-2}, \quad n=2,3, \ldots,
$$

and

$$
\lambda_{f}(0) \geq \lambda_{0}(M)= \begin{cases}\frac{\sqrt{2}}{\sqrt{M^{2}-1}+\sqrt{M^{2}+1}}, & 1 \leq M \leq M_{0}=\frac{\pi}{2 \sqrt[4]{2 \pi^{2}-16}}, \\ \frac{\pi}{4 M}, & M>M_{0}=\frac{\pi}{2 \sqrt[4]{2 \pi^{2}-16}} \approx 1.1296,\end{cases}
$$

where $\lambda_{f}(z)=|| f_{z}(z)|-| f_{\bar{z}}(z)||$. 
Theorem 9. Let $G(z)=\sum_{n=1}^{p-1} \gamma_{n} r^{2 n} F(z)+H(z)$, where $F(z)$ and $H(z)$ are complex-valued harmonic mappings in the unit disk $U$ and $\gamma_{n}, n=1, \ldots, p-1$ $\left(\gamma_{1}^{2}+\cdots+\gamma_{p-1}^{2} \neq 0\right)$ are constants, be a p-harmonic mapping of the unit disk $U$ where $z=r e^{i \theta}$. Assume that $G(0)=H(0)=J_{G}(0)-1=0$ and $|F|$ is bounded by $M_{1}$ while $|H|$ is bounded by $M_{2}$. Then there is a constant $0<\rho_{1}<1$ so that $G$ is univalent in $|z|<\rho_{1}$, where $\rho_{1}$ is the minimum positive root of the following equation

$$
\begin{aligned}
& \lambda_{0}\left(M_{2}\right)-2 \rho_{1} M_{1} \sum_{n=1}^{p-1} n\left|\gamma_{n}\right| \rho_{1}^{2 n-2}-\frac{2 M_{1} \rho_{1}^{2}}{\left(1-\rho_{1}\right)^{2}} \sum_{n=1}^{p-1}\left|\gamma_{n}\right| \rho_{1}^{2 n} \\
& -\sqrt{2 M_{2}^{2}-2}\left(\frac{1}{\left(1-\rho_{1}\right)^{2}}-1\right)=0,
\end{aligned}
$$

and $G\left(U_{\rho_{1}}\right)$ contains a disk $U_{R_{1}}$ where

$$
R_{1}=\lambda_{0}\left(M_{2}\right) \rho_{1}-2 M_{1} \frac{\sum_{n=1}^{p-1}\left|\gamma_{n}\right| \rho_{1}^{2 n+1}}{1-\rho_{1}}-2 M_{2} \frac{\rho_{1}^{2}}{1-\rho_{1}},
$$

and $\lambda_{0}\left(M_{2}\right)$ is as defined in Lemma 2.

Proof. Assume $G(z)=\sum_{n=1}^{p-1} \lambda_{n} r^{2 n} F(z)+H(z)$ satisfies the hypothesis of this theorem, where

$$
\begin{aligned}
& F(z)=F_{1}(z)+\overline{F_{2}(z)}=\sum_{n=0}^{\infty} a_{n} z^{n}+\overline{\sum_{n=0}^{\infty} b_{n} z^{n}} \\
& H(z)=H_{1}(z)+\overline{H_{2}(z)}=\sum_{n=1}^{\infty} c_{n} z^{n}+\overline{\sum_{n=1}^{\infty} d_{n} z^{n}}
\end{aligned}
$$

Here $F_{1}, F_{2}, H_{1}$ and $H_{2}$ are analytic in $D$. Note that

$$
\begin{aligned}
G_{z} & =\sum_{n=1}^{p-1}\left[n \gamma_{n} r^{2 n-2} \bar{z} F+\gamma_{n} r^{2 n} F_{z}\right]+H_{z}, \\
G_{\bar{z}} & =\sum_{n=1}^{p-1}\left[n \gamma_{n} r^{2 n-2} z F+\gamma_{n} r^{2 n} F_{\bar{z}}\right]+H_{\bar{z}} .
\end{aligned}
$$

For fixed $0<\rho<1$, choose $z_{1}, z_{2}$ with $z_{1} \neq z_{2},\left|z_{1}\right|<\rho$ and $\left|z_{2}\right|<\rho$. Then

$$
\begin{aligned}
G\left(z_{1}\right)-G\left(z_{2}\right)= & \int_{\left[z_{1}, z_{2}\right]} G_{z}(z) d z+G_{\bar{z}}(z) d \bar{z} \\
= & \int_{\left[z_{1}, z_{2}\right]}\left[\left(\sum_{n=1}^{p-1} n \gamma_{n} r^{2 n-2} \bar{z} F+\sum_{n=1}^{p-1} \gamma_{n} r^{2 n} F_{1}^{\prime}+H_{1}^{\prime}\right) d z\right. \\
& \left.+\left(\sum_{n=1}^{p-1} n \gamma_{n} r^{2 n-2} z F+\sum_{n=1}^{p-1} \gamma_{n} r^{2 n} F_{2}^{\prime}+\overline{H_{2}^{\prime}}\right) d \bar{z}\right]
\end{aligned}
$$


where $\left[z_{1}, z_{2}\right]$ is the line-segment joining $z_{1}$ with $z_{2}$. We have

$$
1=J_{G}(0)=\left|G_{z}(0)\right|^{2}-\left|G_{\bar{z}}(0)\right|^{2}=\left|H_{z}(0)\right|^{2}-\left|H_{\bar{z}}(0)\right|^{2}=J_{H}(0) .
$$

It follows by Lemma 2 that $\lambda_{H}(0) \geq \lambda_{0}\left(M_{2}\right)$. By the hypothesis of Theorem 9 and Lemmas 1 and 2, we have

$$
\left|a_{n}\right|+\left|b_{n}\right| \leq 2 M_{1} \quad(n=1,2, \ldots), \quad\left|c_{n}\right|+\left|d_{n}\right| \leq \sqrt{2 M_{2}-2} \quad(n=2,3, \ldots) .
$$

As in Theorem 8, by using the triangle inequality, we get

$$
\begin{aligned}
& \left|G\left(z_{1}\right)-G\left(z_{2}\right)\right| \geq\left|\int_{\left[z_{1}, z_{2}\right]}\left(H_{z}(0) d z+H_{\bar{z}}(0) d \bar{z}\right)\right| \\
& -\mid \int_{\left[z_{1}, z_{2}\right]} \sum_{n=1}^{p-1} n \gamma_{n} r^{2 n-2} F(z)(\bar{z} d z+z d \bar{z})+\int \sum_{\left[z_{1}, z_{2}\right]} \sum_{n=1}^{p-1} \gamma_{n} r^{2 n}\left(F_{z}(z) d z+F_{\bar{z}}(z) d \bar{z}\right) \\
& +\int_{\left[z_{1}, z_{2}\right]}\left(H_{z}(z)-H_{z}(0)\right) d z+\left(H_{\bar{z}}(z)-H_{\bar{z}}(0)\right) d \bar{z} \mid \\
& =\left|\int_{\left[z_{1}, z_{2}\right]}\left(H_{1}^{\prime}(0) d z+\overline{H_{2}^{\prime}(0)} d \bar{z}\right)\right|-\mid \int_{\left[z_{1}, z_{2}\right]} \sum_{n=1}^{p-1} n \gamma_{n} r^{2 n-2} F(z)(\bar{z} d z+z d \bar{z}) \\
& +\int_{\left[z_{1}, z_{2}\right]} \sum_{n=1}^{p-1} \gamma_{n} r^{2 n}\left(F_{1}^{\prime}(z) d z+\overline{F_{2}^{\prime}(z)} d \bar{z}\right) \\
& +\int_{\left[z_{1}, z_{2}\right]}\left(H_{1}^{\prime}(z)-H_{1}^{\prime}(0)\right) d z+\left(\overline{H_{2}^{\prime}(z)}-\overline{H_{2}^{\prime}(0)}\right) d \bar{z} \mid \\
& \geq\left|z_{2}-z_{1}\right|\left(\lambda_{H}(0)-2 \rho M_{1} \sum_{n=1}^{p-1} n\left|\gamma_{n}\right| \rho^{2 n-2}\right. \\
& \left.-\sum_{n=1}^{p-1}\left|\gamma_{n}\right| \rho^{2 n} \sum_{n=1}^{\infty}\left(\left|a_{n}\right|+\left|b_{n}\right|\right) n \rho^{n-1}-\sum_{n=2}^{\infty}\left(\left|c_{n}\right|+\left|d_{n}\right|\right) n \rho^{n-1}\right) \\
& \geq\left|z_{2}-z_{1}\right|\left(\lambda_{0}\left(M_{2}\right)-2 \rho M_{1} \sum_{n=1}^{p-1} n\left|\gamma_{n}\right| \rho^{2 n-2}-2 M_{1} \sum_{n=1}^{p-1}\left|\gamma_{n}\right| \rho^{2 n} \sum_{n=1}^{\infty} n \rho^{n-1}\right. \\
& \left.-\sqrt{2 M_{2}^{2}-2} \sum_{n=2}^{\infty} n \rho^{n-1}\right) \\
& \geq\left|z_{2}-z_{1}\right|\left(\lambda_{0}\left(M_{2}\right)-2 \rho M_{1} \sum_{n=1}^{p-1} n\left|\gamma_{n}\right| \rho^{2 n-2}-\frac{2 M_{1} \rho^{2}}{(1-\rho)^{2}} \sum_{n=1}^{p-1}\left|\gamma_{n}\right| \rho^{2 n}\right. \\
& \left.-\sqrt{2 M_{2}^{2}-2}\left(\frac{1}{(1-\rho)^{2}}-1\right)\right) \text {. }
\end{aligned}
$$


In the last inequality, the function $\Lambda(\rho)$, defined by

$$
\begin{aligned}
\Lambda(\rho)= & \lambda_{0}\left(M_{2}\right)-2 \rho M_{1} \sum_{n=1}^{p-1} n\left|\gamma_{n}\right| \rho^{2 n-2}-\frac{2 M_{1} \rho^{2}}{(1-\rho)^{2}} \sum_{n=1}^{p-1}\left|\gamma_{n}\right| \rho^{2 n} \\
& -\sqrt{2 M_{2}^{2}-2}\left(\frac{1}{(1-\rho)^{2}}-1\right)
\end{aligned}
$$

is a decreasing function of $\rho$ on the interval $(0,1)$. Also, $\lim _{\rho \rightarrow 0^{+}} \Lambda(\rho)=$ $\lambda_{0}\left(M_{2}\right)>0$ and $\lim _{\rho \rightarrow 1^{-}} \Lambda(\rho)=-\infty$. Thus there is a unique root $\rho_{1} \in(0,1)$ of the function $\Lambda(\rho)$. This shows that $\left|G\left(z_{1}\right)-G\left(z_{2}\right)\right| \geq\left|z_{2}-z_{1}\right| \Lambda(\rho)>0$ for any two distinct points $z_{1}, z_{2} \in|z|<\rho_{1}$, which proves the univalency of $f$ in the disk $U_{\rho_{1}}$. Following a similar argument as in Theorem 8: for any $z$ with $|z|=\rho_{1}$ we have

$$
\begin{aligned}
|G(z)| & \geq\left|c_{1} z+d_{1} \bar{z}\right|-\sum_{n=1}^{p-1}\left|\gamma_{n}\right| \rho_{1}^{2 n}\left|\sum_{n=1}^{\infty}\left(a_{n} z^{n}+b_{n} \bar{z}^{n}\right)\right|-\left|\sum_{n=2}^{\infty}\left(c_{n} z^{n}+d_{n} \bar{z}^{n}\right)\right| \\
& \geq \lambda_{0}\left(M_{2}\right) \rho_{1}-\sum_{n=1}^{p-1}\left|\gamma_{n}\right| \rho_{1}^{2 n} \frac{2 M_{1} \rho_{1}}{1-\rho_{1}}-\frac{2 M_{2} \rho_{1}^{2}}{1-\rho_{1}} .
\end{aligned}
$$

\section{References}

[1] Z. Abdulhadi and Y. Muhanna. Landau's theorem for biharmonic mappings. J. Math. Anal. Appl., 338:705-709, 2008. http://dx.doi.org/10.1016/j.jmaa.2007.05.065.

[2] Z. Abdulhadi, Y. Muhanna and S. Khuri. On the univalence of the logbiharmonic mappings. J. Math. Anal. Appl., 289(2):629-638, 2004. http://dx.doi.org/10.1016/j.jmaa.2003.08.051.

[3] Z. Abdulhadi, Y. Muhanna and S. Khuri. On univalent solutions of the biharmonic equation. J. Inequal. Appl., 2005(5):469-478, 2005. http://dx.doi.org/10.1155/JIA.2005.469.

[4] Z. Abdulhadi, Y. Muhanna and S. Khuri. On some properties of solutions of the biharmonic equation. Appl. Math. Comput., 177(1):346-351, 2006. http://dx.doi.org/10.1016/j.amc.2005.11.013.

[5] Z. Abdulhadi, Y. Muhanna and S. Khuri. Logharmonicity and logbiharmonicity properties of a nonlinear complex operator. Int. J. Nonlinear Oper. Theory Appl., 2(1):73-82, 2007.

[6] Sh. Chen, S. Ponnusamy and X. Wang. Compositions of harmonic mappings and biharmonic mappings. Belg. Math. Soc. Simon Stevin, 17(4):693-704, 2010.

[7] Sh. Chen, S. Ponnusamy and X. Wang. Bloch constant and Landau's theorem for planar $p$-harmonic mappings. J. Math. Anal. Appl., 373:102-110, 2011. http://dx.doi.org/10.1016/j.jmaa.2010.06.025.

[8] Sh. Chen, S. Ponnusamy and X. Wang. Landau's theorem for $p$-harmonic mappings in several variables. Ann. Polon. Math., 103:67-87, 2012. http://dx.doi.org/10.4064/ap103-1-6.

[9] J.G. Clunie and T. Sheil-Small. Harmonic univalent functions. Ann. Acad. Sci. Fenn. Ser. A I, 9:3-25, 1984. 
[10] P. Duren. Univalent Functions. Springer-Verlag, New York, 1983.

[11] P. Duren. Harmonic Mappings in the Plane. Cambridge University Press, 2004.

[12] S.A. Khuri. Biorthogonal series solution of stokes flow problems in sectorial regions. SIAM J. Appl. Math., 56:19-39, 1996. http://dx.doi.org/10.1137/0156002.

[13] S.A. Khuri. On the properties of a class of log-biharmonic functions. Appl. Math. Lett., 24(7):1142-1148, 2011. http://dx.doi.org/10.1016/j.aml.2011.01.040.

[14] P. Li, S. Ponnusamy and X. Wang. Some properties of planar $p$-harmonic and log-p-harmonic mappings. Bull. Malays. Math. Sci. Soc. (2), 2012. to appear

[15] P. Li and X. Wang. Landau's theorem for log-p-harmonic mappings. Appl. Math. Comput., 218(9):4806-4812, 2012.

[16] M.S. Liu. Landau's theorems for biharmonic mappings. Complex Var. Elliptic Equ., 53(9):843-855, 2008. http://dx.doi.org/10.1080/17476930802166337.

[17] Q. Luo and X. Wang. The starlikeness, convexity, covering theorem and extreme points of $p$-harmonic mappings. Bull. Iranian Math. Soc., 2012. to appear

[18] St. Ruscheweyh and L. Salinas. On the preservation of direction-convexity and the Goodman-Saff conjecture. Ann. Acad. Sci. Fenn. Ser. A. I, 14:63-73, 1989. 